\title{
Continuous Blood Glucose Analysis in Vitro and in Vivo
}

\author{
A. M. Albisser, J. Ellman, A. Hanna, Y. Goriya, and H. Minuk \\ The Hospital for Sick Children, Toronto, Canada
}

Summary. A practical method for continuous blood glucose analysis in vivo is described. Using an interference-free enzyme reagent in a modified AutoAnalyzer whole blood glucose concentration can be monitored continuously and interpreted in terms of the actual plasma glucose concentration. The method uses a novel technique for preheating the sample diluent without introducing additional time delays, consumes whole blood at a rate of $0.05 \mathrm{ml} / \mathrm{min}$, and demonstrates a transport delay of $148 \mathrm{~s}$. Other improvements include an average baseline drift of $-0.11 \mathrm{mg} / \mathrm{dl} / \mathrm{h}$ and a mean change in sensitivity of $-0.4 \%$ after $8.5 \mathrm{~h}$. In vitro glucose recovery studies comparing whole blood to the corresponding plasma samples show the method is precise $(101.3 \pm 0.7 \%)$, linearly proportional (slope of $1.007 \pm 0.012$ ) and highly correlated $(>0.998)$ over the range of 0 to $500 \mathrm{mg} / \mathrm{dl}$, with reference to a Beckman glucose analyzer. In vivo applications are presented to show that this method is suitable for use in systems such as the 'artificial endocrine pancreas'.

Key words: Continuous glucose analysis, artificial pancreas, glucose oxidase, whole blood glucose monitoring.

Continuous blood glucose monitoring has been used to advantage in many clinical studies [1-10]; when frequent sampling is essential this is the preferred technique [8-11].

The methodology described here uses a stable enzymic reagent which is specific to $\beta$-dextrose (glucose) $[12-15]$ while being more sensitive and at the same time far less subject to known interferences [14-17] than previously reported methods $[8,11$,
13]. It has been implemented to monitor glycaemia in both diabetic and non-diabetic patients admitted to the Clinical Investigation Unit of the Toronto General Hospital for metabolic and exercise studies. It serves as the glucose sensing component of two 'artificial endocrine pancreas' instruments one of which is used to control glycaemia with meals at the bedside while the other has been applied in the operating room to 'clamp' glycaemia before, during and after surgery for an insulinoma [10].

\section{Materials and Methods}

Analyzers: The whole blood glucose analyzer was assembled from AutoAnalyzer I components (Technicon Instruments Corp., Tarrytown, N. Y.) which included a peristaltic pump, a 6 " dialyzer using a type $\mathrm{C}$ membrane, a recorder and a colorimeter with a $1.5 \mathrm{~cm}$ flow cell and a pair of $505 \mathrm{~nm}$ interference filters in the optical paths. Figure 1 shows the flow diagram. To minimize the transport delay all interconnections between these components were made as short as physically possible while using the usual 'transmission' tubing $(1 / 16 \times 1 / 8$ inch $)$. Saline diluent for the whole-blood samples was preheated in the thermostated water bath to $37^{\circ} \mathrm{C}$ in a mixing coil and then air-segmented prior to the addition of the whole blood stream. This novel technique [13] eliminates the time delay usually needed for sample and diluent heating prior to dialysis in continuous flow analyzers.

A Beckman Glucose Analyzer (Beckman Instruments, Fullerton, Calif.) was used. Its characteristics compared to reference methods have been previously published [18].

Blood Withdrawal: Blood was withdrawn continuously by means of an indwelling double-lumen catheter (DLC) [19] placed percutaneously in a superficial vein of the forearm for human experiments; in dogs the same catheter was sited in an external jugular vein. The DLC enabled withdrawal of whole blood from the subject without systemic anticoagulation, because heparin was delivered through the outer lumen to the very tip of the DLC where it mixed with the blood as it was drawn into the inner lumen. The blood was diluted approximately $1: 1$ with a solution of heparin and $0.154 \mathrm{~mol} / 1$ saline drawn from a sterile reservoir through a standard administration set by a sterile pump-tube, which was part of the DLC adaptor assembly (Abjad Industries, Mississauga, 


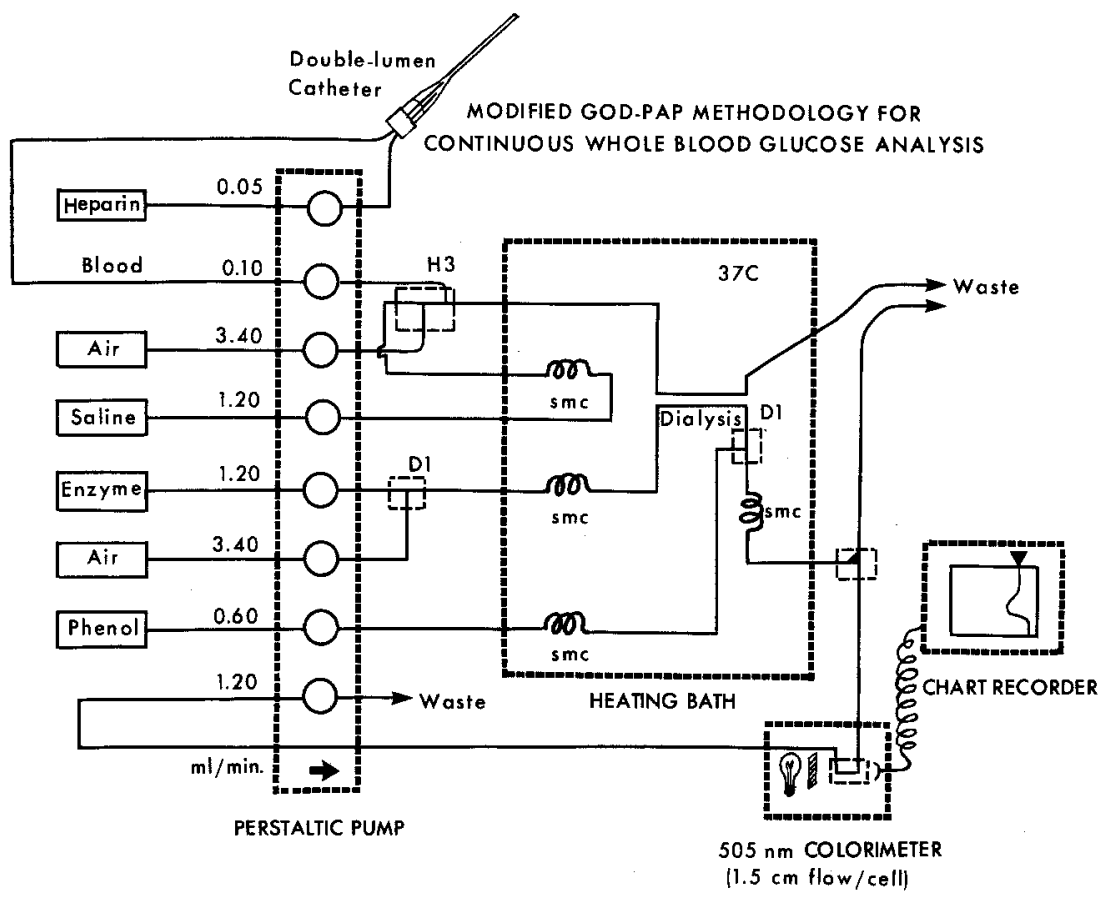

Fig. 1. Flow diagram of an improved method for continuous whole blood glucose analysis. $\mathrm{smc}=$ regular bore single length mixing coil
Ont.). The precise dilution ratio depends on the actual flow rates of the blood-sampling and heparin-delivery pump tubes. For a given tube the delivery rate is constant, but different tubes of the same type will deliver at rates that can differ by as much as $\pm 40 \%$ of their nominal value. The double-lumen catheter adaptor is introduced into a standard 18 or 20 gauge intravenous cannula (Medicut Aloe Medical, St. Louis, Miss.). Blood withdrawal is nominally at $0.05 \mathrm{ml} / \mathrm{min}$ (Figure 1 ).

Reagents: The reagent for glucose analysis used a buffered enzyme (GOD-PAP, Cat. \# 124001, Boehringer-Mannheim, New York, N.Y.) prepared in $0.154 \mathrm{~mol} / 1$ saline. Also, $1 \mathrm{ml}$ of wetting agent (Tween ${ }^{\circledR}$ ) was added to the buffer-enzyme solution and well mixed before being transferred into dark plastic reagent bottles. This solution is stable for 5 weeks at 2 to $8^{\circ} \mathrm{C}$ and for 3 weeks at +15 to $25^{\circ} \mathrm{C}$. Reagent, when not in use, is normally stored in the refrigerator at $4^{\circ} \mathrm{C}$.

The phenol solution was prepared by adding $8 \mathrm{ml}$ of the phenol solution provided to $1000 \mathrm{ml}$ of $0.154 \mathrm{~mol} / 1$ saline and $0.5 \mathrm{ml}$ of the wetting agent. This reagent is stable for 6 months at 15 to $25^{\circ} \mathrm{C}$.

The sterile heparin-saline diluent is prepared fresh at the bedside aseptically by adding 10,000 units of heparin to a $250 \mathrm{ml}$ bag of $0.154 \mathrm{~mol} / 1$ saline.

Sample Preparation: To establish and compare the sensitivity and linearity of this method in vitro, glucose recovery studies were done using the AutoAnalyzer on aqueous standards and whole blood samples. For comparison these recovery studies were also done in the same aqueous samples and the corresponding plasma samples using the Beckman glucose analyzer. Samples for glucose recovery were prepared according to the following steps:

1 . Working glucose standards of $0,100,200,300,400$ and $500 \mathrm{mg} / \mathrm{dl}$ were prepared by adding weighed amounts of anhydrous dextrose to $0.154 \mathrm{~mol} / 1$ saline containing $40 \mathrm{U} / \mathrm{ml}$ of heparin and allowed sufficient time for complete mutarotation.

2. Seven $\mathrm{ml}$ of whole blood was withdrawn from each of 5 healthy subjects. A volume of $500 \mu \mathrm{l}$ of each of the five working standard solutions was then mixed with an equal volume of the whole blood from each subject. This simulates the in-vivo situation when the DLC is used.

3. The remaining blood was then centrifuged and $500 \mu \mathrm{l}$ aliquots of the separated plasma were added to equal volumes of the working glucose standards.

Statistical Methods: Standard statistical comparisons at the 5\% level of significance and linear regression analyses were done.

Glucose Analysis: The Beckman glucose analyzer was initially calibrated according to the instructions of the manufacturer using the aqueous $150 \mathrm{mg} / \mathrm{dl}$ glucose standard provided and the calibration rechecked every 15 minutes to correct for the slow sensitivity changes that occurred. Only the saline-diluted and plasma-diluted samples were assayed in the Beckman glucose analyser (this instrument is not capable of glucose analysis in whole blood samples) and the results obtained are reported as the mean of replicates.

With the AutoAnalyzer all glucose concentrations were computed in the usual way from the data in Table 1.

Bedside Calibration: At the bedside the analyzer must be calibrated so that the readings obtained on whole blood correspond to those values which would be obtained if serial samples were taken for plasma glucose analysis. This is readily done at the start of the study, when an undiluted sample of blood obtained by venepuncture can be used for immediate plasma glucose analysis in an independent laboratory glucose analyzer. This plasma glucose level and the pen deflection of the continuously monitored whole blood glucose record to which it corresponds define a calibration datum point. The line drawn through the baseline recorder deflection at zero glucose and passing through this point calibrates the analyzer. In this way the continuously measured whole blood glucose values obtained for the patient correspond to the actual plasma concentration of glucose at all levels of glycaemia.

Other Characteristics of the In Vivo Methodology: The three most important characteristics of a glucose analyzer used for continuous in vivo blood glucose analysis are its time delay, baseline drift rate 
Table 1. Glucose recovery from the aqueous standards

\begin{tabular}{|c|c|c|c|c|c|c|c|c|}
\hline \multicolumn{2}{|l|}{ Method } & \multicolumn{4}{|c|}{$\begin{array}{l}\text { Glucose concentration } \\
\mathrm{mg} / \mathrm{dl}\end{array}$} & \multicolumn{2}{|r|}{ Slope \pm Sem } & \multirow{2}{*}{$\begin{array}{l}\begin{array}{l}\text { Intercept } \pm \text { Sem } \\
\mathrm{mg} / \mathrm{dl}\end{array} \\
0 \pm 0.0\end{array}$} \\
\hline Expected & 0 & 100 & 200 & 300 & 400 & 500 & $1.000 \pm 0.0$ & \\
\hline Beckman $^{a}$ & 0 & $\begin{array}{l}100 \\
\operatorname{Rec} \\
\%\end{array}$ & $\begin{array}{l}203 \\
\text { deflec }\end{array}$ & 301 & 400 & 502 & $1.002 \pm 0.003$ & $0 \pm 1$ \\
\hline AutoAnalyzer & 95 & 53 & 30 & 17 & 9 & 5 & $(-2.557 \pm 0.022) \times 10^{-3}$ & $95 \pm 1$ \\
\hline
\end{tabular}

a Calibrated to $150 \pm 3 \mathrm{mg} / \mathrm{dl}$ using Beckman aqueous standard solution

and sensitivity change. Delay is the time taken for the recorder to begin its deflection after a step change in glucose concentration is sampled by the DLC.

Baseline drift is the change in the recorder deflection observed when a sample with zero glucose concentration is analyzed some time after the initial baseline adjustment and the result expressed as a rate in $\mathrm{mg} / \mathrm{dl} / \mathrm{h}$. Sensitivity change is defined as the total change in recorder deflection observed from the beginning to the end of an experiment when the $150 \mathrm{mg} / \mathrm{dl}$ standard is analyzed.

\section{Results}

Glucose Recovery from Aqueous Standards: For the two methods of analysis the expected and measured values corresponding to the glucose concentrations of the working standards are presented in Table 1.

Glucose Recovery in Whole Blood Using the AutoAnalyzer: With the AutoAnalyzer glucose recovery from the whole blood samples of the 5 subjects ranged from $100.9 \pm 0.3 \%$ to $101.8 \pm 0.7 \%$ with a mean recovery of $101.3 \pm 0.7 \%$, which is not significantly different from the expected value of $100.0 \%$ as shown in Table 2 .

Glucose Recovery in Plasma Using the Beckman Analyzer: With the Beckman glucose analyzer the recovery of glucose from the corresponding plasma samples ranged from $93.0 \pm 3.0 \%$ to $99.8 \pm 3.2 \%$, with a mean of $97.0 \pm 1.9 \%$, which is not significantly different from $100.0 \%$ as shown in Table 3 .

In Vitro Comparison of the Methods: The graph in Figure 2 compares the two methods and demonstrates that whole blood glucose levels measured by the AutoAnalyzer are linearly proportional to the corresponding 'corrected' plasma glucose levels measured by the Beckman glucose analyzer. Each plasma glucose level was corrected by dividing by the slope of the recovery curve for that subject (taken from Table 3) in order to obtain the equivalent plasma glycaemia that would have been measured if the recovery for each subject were $100 \%$. When this is done the slope of the regression line is $1.007 \pm$ 0.012 , the correlation coefficient is 0.999 , and the line extrapolates through the origin. The low recov-
Table 2. Glucose recovery in whole blood samples using the AutoAnalyzer

\begin{tabular}{lllllllll}
\hline $\begin{array}{l}\text { Sub- } \\
\text { ject }\end{array}$ & \multicolumn{4}{l}{$\begin{array}{l}\text { Increment in blood glucose } \\
\text { concentration mg/dl }\end{array}$} & Slope \pm Sem & $\begin{array}{l}\text { Inter- } \\
\text { cept } \pm \\
\text { Sem } \\
\mathrm{mg} / \mathrm{dl}\end{array}$ \\
& $\overline{0}$ & 50 & 100 & 150 & 200 & 250 & & \\
\hline 1 & 41 & 90 & 141 & 190 & 243 & 294 & $1.013 \pm 0.006$ & $40 \pm 1$ \\
2 & 42 & 92 & 143 & 192 & 247 & 294 & $1.014 \pm 0.008$ & $42 \pm 1$ \\
3 & 38 & 92 & 141 & 190 & 243 & 294 & $1.018 \pm 0.007$ & $39 \pm 1$ \\
4 & 37 & 87 & 137 & 187 & 239 & 289 & $1.009 \pm 0.003$ & $37 \pm 1$ \\
5 & 46 & 95 & 145 & 195 & 247 & 299 & $1.012 \pm 0.006$ & $45 \pm 1$ \\
Mean & 40 & 91 & 142 & 192 & 243 & 294 & $1.013 \pm 0.007$ & $40 \pm 1$ \\
\hline
\end{tabular}

Table 3. Glucose recovery in plasma samples using the Beckman Analyzer

\begin{tabular}{llllllll}
\hline $\begin{array}{l}\text { Sub- } \\
\text { ject }\end{array}$ & $\begin{array}{l}\text { Increment in blood } \\
\text { glucose concentration } \\
\text { mg/dl }\end{array}$ & & Slope \pm Sem & $\begin{array}{l}\text { Intercept } \pm \\
\text { Sem } \\
\mathrm{mg} / \mathrm{dl}\end{array}$ \\
\cline { 2 - 8 } & 0 & 50 & 100 & 150 & 200 & & \\
\hline 1 & 41 & 87 & 139 & 188 & - & $0.986 \pm 0.016$ & $40 \pm 1$ \\
2 & 42 & 94 & 135 & 187 & 245 & $0.998 \pm 0.032$ & $41 \pm 4$ \\
3 & 40 & 88 & 125 & 177 & 228 & $0.930 \pm 0.030$ & $39 \pm 4$ \\
4 & 34 & 91 & 130 & 181 & - & $0.960 \pm 0.046$ & $37 \pm 4$ \\
5 & 49 & 94 & 141 & 188 & 243 & $0.964 \pm 0.021$ & $47 \pm 3$ \\
Mean & 40 & 89 & 137 & 186 & 234 & $0.970 \pm 0.019$ & $40 \pm 2$ \\
\hline
\end{tabular}

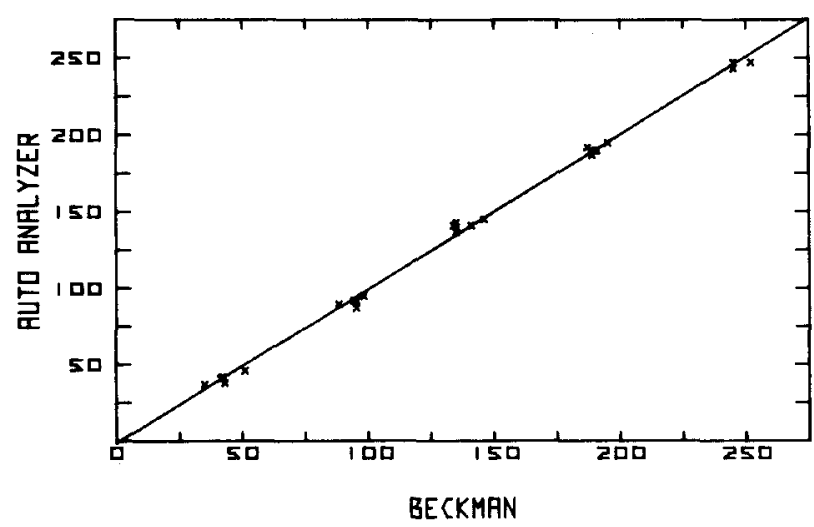

Fig. 2. Dextrose recovery $(\mathrm{mg} / \mathrm{dl})$ in whole blood using an AutoAnalyzer compared to dextrose recovery $(\mathrm{mg} / \mathrm{dl})$ in plasma using a Beckman Glucose Analyzer. The line representing the agreement between the two methods has a slope of $1.007 \pm 0.012$ and extrapolates through the origin. Whole blood (and plasma) samples are from 5 healthy subjects. Data from two subjects overlap 


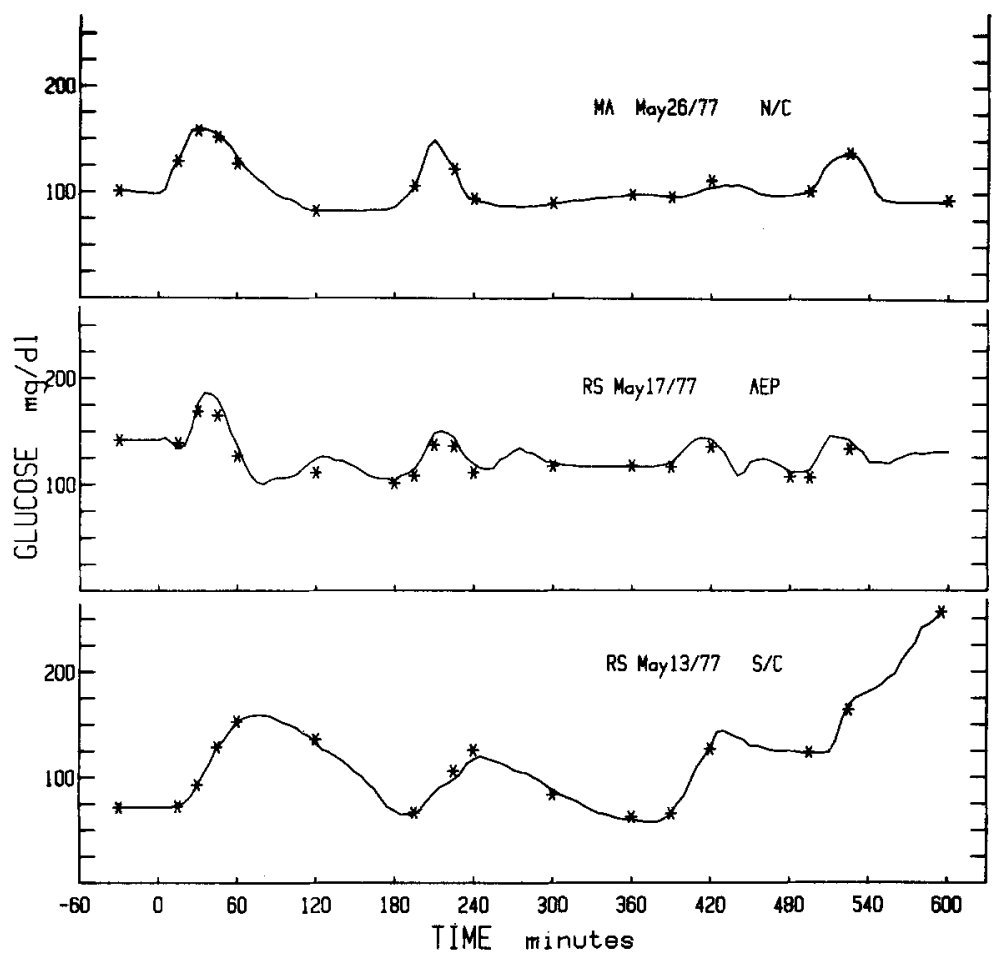

Fig. 3. Continuous whole blood glucose concentrations using the AutoAnalyzer (solid lines) and discrete plasma glucose concentrations using the Beckman (asterisks). Breakfast at 0 , lunch at 180 , snack at 360 and dinner at 480 minutes. Upper panel: normal subject - Middle panel: juvenile diabetic treated with the artificial endocrine pancreas Lower panel: same juvenile diabetic subject treated with subcutaneous insulin at -30 minutes ery of the Beckman analyzer on the plasma samples is due to the use of an air displacement micro-pipette [20].

In Vivo Comparison of the Methods: Examples of the continuously monitored in-vivo whole blood glucose levels for two different subjects studied on three separate occasions are shown in Figure 3. There is clearly excellent agreement at all glucose concentrations between the continuous in vivo record and the plasma glucose levels which were measured in independently drawn samples at the frequent intervals indicated.

Delay, Sensitivity Change and Baseline Drift Rate: Table 4 presents the data from 6 experiments showing the time delay, baseline drift rate and sensitivity change observed with the application of this continuous in vivo methodology.

\section{Discussion}

The improved method described in this paper enables continuous in-vivo whole blood glucose analysis at the bedside using a modified AutoAnalyzer. It has been applied as the blood glucose sensor in studies involving glycaemic regulation using an artificial pancreas in diabetics subjected to caloric challenge $[21]$, to exercise $[22,23]$ and during labour and
Table 4. Characteristics of the in vivo continuous blood glucose method

\begin{tabular}{llllc}
\hline $\begin{array}{l}\text { Study } \\
\#\end{array}$ & $\begin{array}{l}\text { Delay } \\
\text { sec }\end{array}$ & $\begin{array}{l}\text { Baseline drift } \\
\text { rate } \\
\mathrm{mg} / \mathrm{dl} / \mathrm{h}\end{array}$ & $\begin{array}{l}\text { Sensitivity } \\
\text { change }^{\mathrm{a}} \\
\%\end{array}$ & $\begin{array}{l}\text { Time } \\
\mathrm{h}\end{array}$ \\
\hline 1 & 150 & +0.26 & 0 & 12.5 \\
2 & 150 & +0.32 & -1.5 & 10 \\
3 & 145 & -0.30 & -1 & 6.5 \\
4 & 143 & -0.50 & +1 & 4 \\
5 & 150 & -0.42 & -0.5 & 10 \\
6 & 149 & -0.0 & -0.5 & 8 \\
Mean & 148 & -0.11 & -0.42 & 8.5 \\
\hline
\end{tabular}

a Change in recorder deflection ( $\%$ of full scale) with the $150 \mathrm{mg} / \mathrm{dl}$ standard over the time period noted

delivery. It has also proven of value in the operating suite as a glucose clamp during pancreatic resection for insulinoma [10]. Through the use of the doublelumen catheter these studies are conducted without the introduction of an anticoagulant into the subjects and no complications of any kind have been encountered during, or reported following, well over 50 studies in humans during the last 2 years. The results so obtained compare exactly to an accepted laboratory technique using a Beckman glucose analyzer. Invitro assessments of both methods demonstrate the linearity and precision of glucose recovery from prepared aqueous glucose standards (Table 1), from human plasma (Table 3) and whole blood (Table 2) 
to which equal volumes of these aqueous standards have been added. The new method precisely recovers glucose over the range of 0 to $500 \mathrm{mg} / \mathrm{dl}$.

The transport delay of this monitoring methodology is $148 \mathrm{~s}$, lying between that of previous methods $270 \mathrm{~s}[8,11]$ and $90 \mathrm{~s}[13]$, respectively. Compared to these, the present method is more stable and also about twice as sensitive, while consuming whole blood at the same rate of $0.05 \mathrm{ml} / \mathrm{min}$. With this increased sensitivity the blood requirements could be reduced. However, this is difficult to realize with consistency because of the aforementioned wide variations in flow-rates obtained with the smaller bore pump-tubes that would be needed for blood sampling. Furthermore, decreasing the flow-rate would increase both the noise and the presently observed $30 \mathrm{~s}$ transport delay through the capillary bore blood sampling tube. The enzyme method [12] used is free from the usual interferences [24-27] even when modified as here described and extended to whole blood.

Routine recalibration after $4-12.5 \mathrm{~h}$ of continuous blood glucose analysis shows a small, variable baseline drift ranging from +0.3 to $-0.5 \mathrm{mg} / \mathrm{dl} / \mathrm{h}$ and no significant reduction in the sensitivity or slope of the calibration curve. Such stability implies a remarkable consistency not only of the reagent but also of the dialyzer and the colorimeter. In this regard the method described is over an order of magnitude better than that of a glucose electrode recently described by others [28]. Reagent is consumed at the rate of only $1.2 \mathrm{ml} / \mathrm{min}$ so that the running cost is currently only about $\$ 1.00$ per $h$.

Offsets about the origin in the curve relating different methods of glucose analysis have been observed by others $[14,15]$. Such offsets do not occur with this improved methodology: the linear regression curve in Figure 2 extrapolates through the origin. Thus this method allows the interpretation of continuously monitored whole blood glucose levels in terms of the accepted plasma glucose values [29]. As shown in Figure 3 samples taken periodically during experiments verify the consistency of this methodology in providing true 'plasma' glucose values.

The technique described in this paper is reliable and stable in terms of drift and sensitivity and has been implemented as part of a clinical apparatus known as an artificial endocrine pancreas which is currently used for studies of the pathophysiology of diabetes.

Acknowledgements. We gratefully acknowledge the help of Mr. G. Skinner and Ms. L. Willms of Boehringer Mannheim Canada in developing the methodology. Some AutoAnalyzer components were kindly provided by Mr. Ted Henderson of Technicon International, Montreal, Quebec. The Beckman glucose analyzer was generously donated by the Toronto and District Branch and the Mothers Auxiliary of the Canadian Diabetic Association.

This study was conducted as a project in the artificial pancreas programme at the Hospital for Sick Children in Toronto and benefitted in part from the support provided by the Juvenile Diabetes Research Foundation, the Juvenile Diabetes Foundation, the Medical Research Council of Canada, and the Pfizer Company of Canada. The support of the Mount Sinai Hospital for A. Hanna is gratefully acknowledged.

\section{References}

1. Weller, C., Linder, M., Macaulay, A., Ferrari, A., Kessler, G.: Continuous in vivo determination of blood glucose in human subjects. Ann. N. Y. Acad. Sci. 87, 658-668 (1960)

2. Brown, G. M., Zachwieja, A., Stancer, H. C.: An improved technique for continuous in vivo analysis of glucose. Clin. Chim. Acta 14, 386-394 (1966)

3. Burns, T. W., Bregant, R., Van Peenan, H. J., Hood, T. E.: Observations on blood glucose concentration of human subjects during continuous sampling. Diabetes 14, 186-193 (1965)

4. Molnar, G. D., Ackerman, E., Rosevear, J.W., Gatewood, O. C., Moxness, K. E.: Continuous blood glucose analysis in ambulatory fed subjects. I. General Methodology. Mayo Clin. Proc. 43, 844-851 (1968)

5. Molnar, G. D., Taylor, W. F., Ho. M. M.: Day-to-day variation of continuously monitored glycaemia: a further measure of diabetic instability. Diabetologia 8, 342-348 (1972)

6. Rosevear, J. W., Pfaff, K. J., Service, F. J., Molnar, D. G., Ackerman, E.: Glucose oxidase method for continuous automated blood glucose determination. Clin. Chem. 15, 680-698 (1969)

7. Spathis, G. S.: Continuous monitoring of blood sugar in brittle diabetics. Diabetologia 6, 586-592 (1970)

8. Albisser, A. M., Leibel, B.S., Ewart, T.G., Davidovac, Z., Botz, C. K., Zingg. W., Schipper, H., Gander, R. E.: Clinical control of diabetes by the artificial endocrine pancreas. Diabetes 23, 397-404 (1974)

9. Marliss, E. B., Murray, F. T., Zinman, B., Nakhooda, A. F., Denoga, A., Leibel, B. S., Albisser, A. M.: Normalisation of glycemia in diabetics during meals with insulin and glucagon delivery by the artificial pancreas. Diabetes 26, 664-672 (1977)

10. Kudlow, J., Albisser, A. M., Angel, A., Langer, B., Yip, C., Zinman, B. and Stokes, E.: Insulinoma resection facilitated by the artificial endocrine pancreas. Diabetes (in press) (1978)

11. Albisser, A. M., Leibel, B.S., Ewart, T. G., Davidovac, Z., Botz, C. K., Zingg, W.: An artificial endocrine pancreas. Diabetes 23, 389-396 (1974)

12. Trinder, P.: Determination of blood glucose using an oxidaseperoxidase system with a non-carcinogenic chromogen. J. Clin. Pathol. 22, 158-161 (1969)

13. Albisser, A. M., Leibel, B. S., Johnson, W., Denoga, A., Botz, C. K. and Marliss, E. B.: An improved technique for the rapid continuous measurement of whole blood glucose, suitable for clinical application in an artificial endocrine pancreas. Med. Progr. Technol. 5, 141-148 (1977)

14. Hawkins, P., Parker, D.: The design, construction and evaluation of an inexpensive analyzer for measuring glucose concentration in serum, plasma and whole blood. Phys. Med. Biol. 18, 570-576 (1973)

15. Holtkamp, H. C., Verhoef, M. J., Leijnse, B.: The difference between the glucose concentrations in plasma and whole blood. Clin. Chim. Acta 59, 41-49 (1975) 
16. McDonald, G. W., Fisher, G. F., Burnham, C. E.: Differences in glucose determinations obtained from plasma or whole blood. Public Health Rep. 79, 515-521 (1964)

17. Miskiewicz, S. J., Arnett, B. B., Simon, G. E.: Evaluation of a glucose oxidase-peroxidase method adapted to the single channel AutoAnalyzer and SMA 12/60. Clin. Chem. 19, 253-257 (1973)

18. Gochman, N., Ryan, W.T., Sterling, R. E., Widdowson, G. M.: Interlaboratory comparison of enzymatic methods for serum glucose determination. Clin. Chem. 21, 356-361 (1975)

19. Gander, R. E., Albisser, A. M., Botz, C. K., Leibel, B. S., Zingg, W.: An all plastic double-lumen catheter for continuous blood sampling. J. A. A. M. I. 9, 187-188 (1975)

20. Medina, F., Cheong, V., Peck, C. and Bensinger, T. A.: Improved method for using eppendorf pipettes for accurate delivery of blood. Clin. Chem. 23, 1188-1189 (1977)

21. Albisser, A. M., Leibel, B.S., Zinman, B., Murray, F.T., Zingg, W., Botz, C. K., Denoga, A., Marliss, E. B.: Studies with an artificial endocrine pancreas. Arch. Intern. Med. 137, 639-649 (1977)

22. Murray, F. T., Zinman, B., McClean, P. A., Denoga, A., Albisser, A. M., Leibel, B. S., Nakhooda, A. F., Stokes, E. F. and Marliss, E. B.: The metabolic response to moderate exercise in diabetic man receiving intravenous and subcutaneous insulin. J. Clin. Endocrinol. Metab. 44, 708-720 (1977)

23. Zinman, B., Murray, F. T., Vranic, M., Albisser, A. M., Leibel, B. S., McClean, P. A. and Marliss, E. B.: Glucoregulation during moderate exercise in insulin treated diabetics. J.Clin. Endocrinol. Metab. 45, 641-652 (1977)
24. Fingerhut, B.: Automated serum glucose levels in uremia. Am. J. Clin. Pathol. 55, 157-160 (1969)

25. Powell, J.B., Djuh, Y.Y.: A comparison of automated methods for glucose analysis in patients with uremia before and after dialysis. Am. J. Clin. Pathol. 56, 8-16 (1971)

26. Carey, R. N., Feldbruegge, D., Westgard, J. O.: Evaluation of the adaptation of the glucose oxidase/peroxidase-3-methyl-2benzothiazolinone hydrazone- $\mathrm{N}, \mathrm{N}$-dimethylaniline procedure to the Technicon "SMA $12 / 20$ ", and comparison with other automated methods for glucose. Clin. Chem. 20, 595-602 (1974)

27. Peterson, J.I., Young, D. S.: Evaluation of the hexokinase/ glucose-6-phosphate dehydrogenase method of determination of glucose in urine. Anal. Biochem. 23, 301-316 (1968)

28. Clarke, W. L., Santiago, J. V.: The characteristics of a new glucose sensor for use in an artificial pancreatic beta cell. Art. Org. 1, 78-82 (1977)

29. Zalme, E., Knowles, H. C.: A plea for plasma sugar. Diabetes 14, 165-166 (1965)

Received: April 10, 1978

and in revised form: June 12, 1978

Dr. A. M. Albisser

Biomedical Research

The Hospital for Sick Children

555 University Avenue

Toronto, Ontario

Canada 